10.1117/2.1200908.1752

\title{
Functional quantum circuit combines photon gates
}

Shigeki Takeuchi, Jeremy O'Brien, Holger Hofmann, Keiji Sasaki, Tomohisa Nagata, and Ryo Okamoto

An optical-quantum-circuit entanglement filter can pass a pair of photons with the desired polarization correlations.

Photons are an important information-conveyance medium for enhanced computers and communications based on quantum mechanics. Filtering multiple photon states is a key capability in quantum-information science and technology, where onequantum binary-digit (one-qubit) filters, or polarizers, have found wide application. Filtering based on the correlation or entanglement of photons requires multi-qubit filters with qubitqubit interactions.

We demonstrate an optical-entanglement filter that passes a pair of photons if they have the desired polarization correlations. ${ }^{1}$ The entangling capability of the filter was verified, distinguishing it from classical ones. Because our entanglement filter acts on photonic qubits, it is promising for quantum technologies, since photons are the logical choice for communication, metrology, and lithography. For example, the filter can be used to create and purify entanglements (see Figure 1), which will be important in realizing quantum relays and repeaters for longdistance communication. Furthermore, the optical quantum circuit is one of the world's largest in terms of both the number of gates and classical interferences. This achievement shows potential for developing a variety of circuits.

Such a device has been proposed for photonic qubits. ${ }^{2}$ However, the technical requirements to build an optical circuit with two ancillary photons and multiple quantum gates, requiring both quantum and classical interferences in several nested interferometers, have been lacking.

We overcame these difficulties by combining two key recent technological approaches, a displaced-Sagnac architecture ${ }^{3}$ and partially polarizing beam splitters (PPBSs). ${ }^{4}$ The PPBSs reflect vertically polarized photons perfectly while transmitting or reflecting horizontally polarized photons with $50 \%$ probability. Replacing all beam splitters with PPBSs, we successfully removed two optical paths. The remaining optical-path

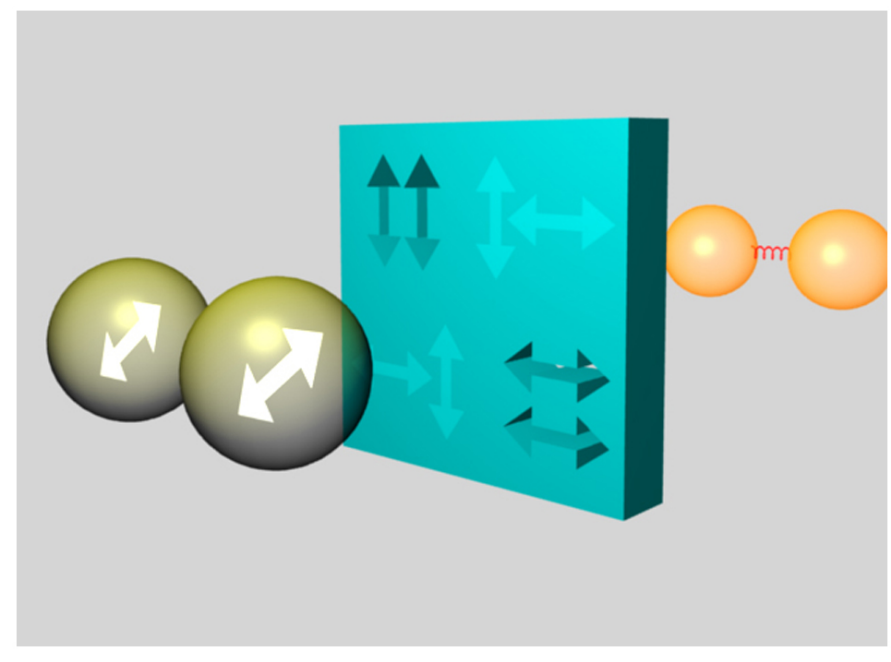

Figure 1. An entanglement filter makes it possible to extract only photon pairs in a state of specific quantum entanglement.

interferometer was realized as a displaced-Sagnac setup (see Figure 2). All four polarization modes of the two input photons passed through all optical components inside the interferometer so the path differences of the four polarization modes were robust against optical-component drifts or vibrations.

First, we checked the essential operation of the filter circuit. We prepared input signal photons in the four combinations of horizontal $(\mathrm{H})$ and vertical $(\mathrm{V})$ polarizations, and observed how the circuit filtered them (see Figure 3). It was clear from experimental data that the photon pairs were transmitted through the filter when the two input photons shared the same polarization (HH or VV). Most were filtered out when the two input photons had different polarizations ( $\mathrm{HV}$ or $\mathrm{VH})$. The fidelity of this process can be defined as the ratio of correctly transmitted to the total number of transmitted photon pairs. For the filter's operation on horizontal and vertical polarizations, the fidelity was 0.80 .

The entanglement filter will be a key element in controlling multi-photon quantum states in applications such as quantum

Continued on next page 


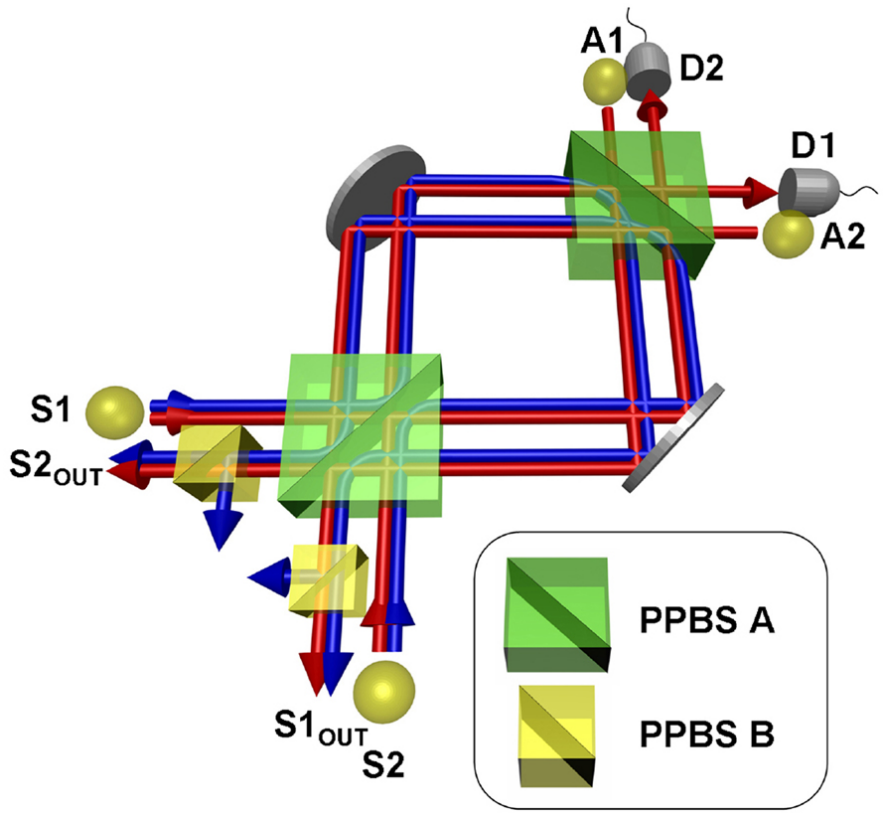

Figure 2. An optical quantum circuit for a nondestructive entanglement filter. The reflectance (transmittance) of partially polarizing beam splitter (PPBS) A (B) for horizontally and vertically polarized photons is 0.5 and 1, respectively. The system includes one superstable opticalpath interferometer that uses a displaced-Sagnac architecture. A1 and $A 2$ are ancillary input photons, while D1 and D2 are two different photon counters. S1 and S2 denote two different signal-photon inputs, and $S 1_{\text {out }}$ and $S 2_{\text {out }}$ are the signal outputs.
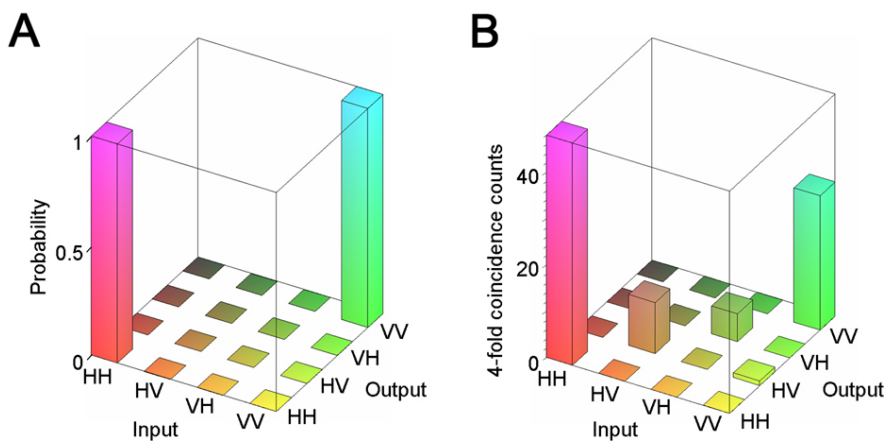

Figure 3. Experimental results. Input signal photons are prepared in horizontal $(H)$ or vertical $(V)$ polarization and measured on an $H / V$ basis. (a) Theoretically predicted probabilities, and (b) four-fold coincidence count rates (counts/800s) of the four detectors for signal and ancillary photons.

communications and information processing. For present performance tests of the quantum-filter circuit, we used threshold detectors to monitor the output state. For applications where the output state cannot be monitored, high-efficiency, numberresolving photon detectors could be used to generate the heralding signals. Such a circuit could be used for on-demand generation of entangled photons or nondestructive entanglement purification. It could be implemented using an integrated waveguide architecture. We are currently using similar techniques to realize different types of quantum circuits for novel future applications.

This work was supported by the Japan Science and Technology Agency, Ministry of Internal Affairs and Communication, Japan Society for the Promotion of Science, 21st Century Centers of Excellence Program, and Special Coordination Funds for Promoting Science and Technology.

\section{Author Information}

\section{Shigeki Takeuchi, Keiji Sasaki, Tomohisa Nagata, and}

\section{Ryo Okamoto}

Research Institute for Electronic Science

Hokkaido University

Sapporo, Japan

and

The Institute of Scientific and Industrial Research

Osaka University

Osaka, Japan

http:/ / www.sanken.osaka-u.ac.jp/labs/qip/index_e.html

\section{Jeremy $O^{\prime}$ Brien}

University of Bristol

Bristol, UK

\section{Holger Hofmann}

Hiroshima University

Higashi-Hiroshima, Japan

References

1. R. Okamoto, J. O'Brien, H. F. Hofmann, T. Nagata, K. Sasaki, and S. Takeuchi, An entanglement filter, Science 323, p. 483, 2009.

2. H. F. Hofmann and S. Takeuchi, Quantum filter for nonlocal polarization properties of photonic qubits, Phys. Rev. Lett. 88, p. 147901, 2009.

3. T. Nagata, R. Okamoto, J. O'Brien, K. Sasaki, and S. Takeuchi, Beating the standard quantum limit with four-entangled protons, Science 316, p. 726, 2007.

4. R. Okamoto, H. F. Hofmann, S. Takeuchi, and K. Sasaki, Demonstration of an optical quantum controlled-NOT gate without path interference, Phys. Rev. Lett. 95, p. 210506,2005 . 